WIENER SLAVISTISCHES JAHRBUCH, Band 52/2006, 201-212

(C) 2007 by Österreichische Akademie der Wissenschaften, Wien

STEF AN S IMONEK

\title{
Ivan Franko in der Wiener „Arbeiter-Zeitung“ (1889-1901) (Addenda et corrigenda)
}

Einen spezifischen Aspekt der reichhaltigen und methodologisch ausdifferenzierten literaturhistorischen Forschungsarbeiten zu Ivan Franko stellt die Aufarbeitung der publizistischen Verbindungen des ukrainischen Autors zu diversen um 1900 in Wien erschienenen Periodika dar, für die Franko teilweise über Jahre hinweg belletristische, essayistische und wissenschaftliche Beiträge in deutscher Sprache verfasst hat; diese Beiträge harren freilich bis zum heutigen Tage einer wirklich vollständigen und systematischen Katalogisierung. Im Lauf der Jahre sind immer wieder Wissenschaftler aus der Ukraine nach Wien gekommen, um unter teilweise beträchtlichem Zeitdruck und finanziellem Aufwand den Reichtum der Wiener Archive u. a. auch unter diesem Aspekt zu sichten. Was läge für einen Wissenschaftler aus Wien nun also näher, als ohne zeitliche Limitierung und ohne nennenswerte Anreisewege die von ukrainischer, deutscher, polnischer und österreichischer Seite her bis dato gewonnenen Resultate zu ergänzen und gegebenenfalls auch zu korrigieren? In diesem Sinne sollen auch die in Folge präsentierten Addenda und Corrigenda nicht als implizit vorgebrachte Kritik an den ergänzten bzw. korrigierten Aufsätzen verstanden werden, sondern vielmehr als Versuch, den gegebenen Forschungsstand noch weiter als bisher zu präzisieren. Daneben möchte diese knappe Darstellung weiter auch belegen, welche Möglichkeiten sich in Wien als Residenzstadt der ehemaligen Donaumonarchie in Bezug auf die Archivforschung in Sachen Franko gerade für die Wiener Slawistik eröffnen. Die intensive und systematische Sammeltätigkeit der großen Wiener Bibliotheken insbesondere auch in den Kronländern der Monarchie bis in das Jahr 1918 resultierte schließlich in einer gewaltigen bibliographischen Schatztruhe, deren Inhalt gerade auf dem Gebiet der österreichisch-slawischen kulturellen Kontakte über weite Strecken erst ansatzweise gesichtet wurde. ${ }^{1}$

${ }^{1}$ Vgl. in Bezug auf Galizien Magocsi 1979/80. 
Die Entscheidung, gerade die ab 1889 in Wien erschienene „Arbeiter-Zeitung“, das publizistische Organ der österreichischen Sozialdemokratie, in Sachen Ivan Franko durchzusehen, lässt sich dabei in gleich mehrfacher Hinsicht begründen. Hier ist zum ersten die wechselseitige, bis hin zu persönlichen Kontakten reichende Wertschätzung zu erwähnen, die Ivan Franko und Viktor Adler, der Gründer der Sozialdemokratischen Partei Österreichs und der „Arbeiter-Zeitung“, füreinander empfunden haben. Diese Wertschätzung von beiden Seiten lässt sich augenscheinlich an verschiedenen Stellen ablesen. So wäre hier wohl der an Franko adressierte Brief Adlers vom 8. Juli 1898 zu erwähnen, der in den 1963 publizierten deutschen Schriften Frankos veröffentlicht wurde. Adler - der Franko hier mit „Verehrter Genosse!" anredet, kommt in diesem Schreiben auf die manipulierten galizischen Landtagswahlen des Jahres $1898 \mathrm{zu}$ sprechen und plädiert für eine politische Vereinigung der radikalen Ruthenen und der Sozialdemokratie, um so gemeinsam mehr Durchschlagskraft zu gewinnen. Am Schluss seines Briefes erkundigt sich Adler nach belletristischen Beiträgen Frankos für den literarischen Teil der „Arbeiter-Zeitung" (Franko 1963: 473 f.) und verweist damit implizit auf die Ergiebigkeit dieser Quelle und die Sinnhaftigkeit, diese nach bis dato unbekannten Beiträgen Frankos durchzusehen.

Nachweisbare Referenzen in beiden Richtungen finden sich freilich schon vor dem Jahre 1898, nämlich im Winter 1896, als Franko in Wien zwei thematisch miteinander verbundene Vorträge zur Lage des galizischen Bauernstandes gehalten hat. Zum zweiten Vortrag vom 14. Dezember, der im Rahmen einer Versammlung des Politischen Volksvereins in Hambergers Saal, Wien V, stattfand, brachte die „Arbeiter-Zeitung" in der Nummer 346 am 16. Dezember einen ausführlichen Bericht. Darin wird auch die auf Frankos Ausführungen folgende Erwiderung Viktor Adlers zitiert, der Franko einen der bedeutendsten Dichter Österreichs sowie „ein großes Herz und einen großen Kopf, der sein ganzes Wirken in den Dienst des Volkes gestellt hat", nennt (346/16.12.1898/6) ${ }^{2}$. In die andere Richtung, nämlich von Franko zu Adler, führt jener autobiographische Abriss, den Franko zu Jahresbeginn 1909 an die Redaktion von „Herders Konversations-Lexikon“ geschickt hat. Hier zählt Franko u. a. auch jene Wiener Bekanntschaften auf, deren Freundschaft seiner Ansicht nach ihm zur Ehre gereicht, und erwähnt dabei auch Viktor Adler gerade in dessen Funktion als Herausgeber der „Arbeiter-Zeitung“ (Franko 1963: 41). Zu einer persönlichen Begegnung zwischen Franko und Adler liegt auch ein schriftliches Zeugnis eines anderen slawischen Schriftstellers vor: Der tschechische Autor Josef Svatopluk Machar, den Franko im Juni 1893 in Wien kennen lernte (vgl. dazu genauer Simonek 1993: 133), berichtet in seinem 1897 veröffentlichten Feuilleton Vlčkoviáda

${ }^{2}$ Zitatnachweis aus der „Arbeiter-Zeitung“ jeweils unter Angabe von Nummer / Erscheinungsdatum / Seitenzahl(en). 
třetí über ein Treffen mit Ivan Franko in einem Wiener Kaffeehaus im Dezember 1896, zu dem später dann auch Viktor Adler dazustieß (Machar 1902: 119).

Als ein weiteres Argument, sich gerade mit der „Arbeiter-Zeitung“ zu beschäftigen, kann die Kontinuität angeführt werden, mit der Franko in diesem Blatt vertreten ist. Aus den verschiedenen Angaben in der Sekundärliteratur ergibt sich der (auch für die vorliegende Arbeit maßgebliche) Zeitraum zwischen den Jahren 1889 und 1901, in dem Texte Frankos in der „Arbeiter-Zeitung“ veröffentlicht wurden. Zu diesem Zweck wurden die Jahrgänge 1889 bis 1899 der „Arbeiter-Zeitung“ (also die Jahrgänge eins bis elf) systematisch durchgesehen und durch einen weiteren Hinweis auf das Jahr 1901 ergänzt. Auch wenn Frankos publizistische Präsenz in anderen Blättern, wie etwa in der Wiener Wochenschrift „Die Zeit“, intensiver und - bedenkt man nur den skandalisierten, Adam Mickiewicz inkriminierenden Aufsatz Ein Dichter des Verrathes (1897) von der Resonanz beim Publikum her bedeutsamer gewesen sein mag, so existiert meines Wissens nach kein anderes deutschsprachiges Periodikum, in dem Franko über dreizehn Jahre hinweg mehr oder weniger kontinuierlich seine Beiträge veröffentlichte (die „Zeit“ erschien in ihrer ursprünglichen Form als Wochenschrift ja lediglich elf Jahre lang von 1894 bis 1904).

Als letzten wesentlichen Grund, gerade das Organ der österreichischen Sozialdemokratie nochmals systematisch auf Beiträge Frankos durchzugehen, sind mehrfach zu beobachtende Widersprüche in der Sekundärliteratur zu erwähnen, die eine anhand der direkten Einsicht in die Quellen erstellbare Korrektur geradezu herausfordern. Wenn im Titel dieses Beitrags nun von Addenda und Corrigenda zum gegenwärtigen Forschungsstand die Rede ist, so muss dieser naturgemäß zunächst eindeutig definiert werden. Neben der fünfzigbändigen Werkausgabe Frankos und dem bereits erwähnten Band seiner deutschsprachigen Texte sowie der 1966 in Kiew erschienenen Bibliographie von Frankos Werken im Zeitraum zwischen 1874 und 1964 (Franko 1966) sind hier in chronologischer Reihenfolge noch folgende Arbeiten zu berücksichtigen: Bohdan Bendzars 1966 veröffentlichter Aufsatz zu den deutschsprachigen publizistischen Werken Frankos (Bendzar 1966), gefolgt von dessen 1969 vorgelegtem Autoreferat zu Frankos deutschsprachigen Werken (Bendzar 1969) ${ }^{3}$, Mykola Zymomrjas materialreiche Ostberliner Dissertation zur Aufnahme der ukrainischen Literatur im deutschen Sprachraum (Zymomrja 1972), Michał Cieślas polnischer Aufsatz zum deutschsprachigen Schaffen Frankos aus dem Jahre 1976 (Cieśla 1976), Jaroslava Pohrebennyks 1988 veröffentliche Übersichtsdarstellung zu den ukrainisch-deutsch-österreichischen literarischen Kontakten im 19. und am Anfang des 20. Jahrhunderts (Pohrebennyk 1988), Günther Wytrzens' 1991 erschienener Aufsatz zum deutschsprachigen Werk Frankos (Wytrzens 1991), Markijan Nahirnyjs Skizze zu Frankos Teilhabe am literaturgeschichtlichen Prozess in

${ }^{3}$ Für die postalische Übermittlung der beiden Arbeiten sei Bohdan Bendzar an dieser Stelle herzlich gedankt. 
Österreich und Deutschland (Nahirnyj 1998) sowie Mykola Zymomrjas Übersicht zur Aufnahme von Frankos Werken in Deutschland aus dem Jahre 1999 (Zymomrja 1999). Textbelege Frankos, die in diesen Arbeiten nicht verzeichnet sind, sollen hier in weiterer Folge als Addenda zum aktuell gegebenen Forschungsstand bezeichnet werden.

Bevor die Rede auf diese Ergänzungen kommt, noch ein Blick auf die Corrigenda, auf nicht korrekte Angaben in der Sekundärliteratur also, die sich durch die Konsultation der Primärquellen recht einfach klären lassen. Hier wäre gleich der erste Textbeleg zu nennen, mit dem Franko 1889 im ersten Jahrgang der neugegründeten „Arbeiter-Zeitung“" vertreten ist, nämlich die Dorfnovelle Die Zigeuner. Laut Pohrebennyk und Nahirnyj erschien der Text am 18. und 25. April 1889 (Pohrebennyk 1988: 252 bzw. Nahirnyj 1998: 803), Zymomrja vermerkt lediglich das Jahr 1889 (Zymomrja 1999: 139). Tatsächlich jedoch erschien der Text in zwei Folgen im „Unterhaltungsblatt“ der „Arbeiter-Zeitung“ am 18. und 25. Oktober (8/18.10. 1889/5-6 bzw. 9/25.10.1889/5-6). Interessant sind an diesem Debüt Frankos im Wiener Blatt weiter noch zwei Dinge: Erstens fehlt hier jegliche erläuternde Vorstellung des neuen Autors, dessen Novelle sich zwischen Texten anerkannter Prosaiker wie etwa Tolstoj, Guy de Maupassant oder Zola wiederfindet, und zweitens zeigt sich bereits hier der schon von Pohrebennyk vermerkte polnische Rezeptionskontext, in dem Frankos Werke stehen (Pohrebennyk 1988: 250). Die Novelle Frankos wurde nämlich nicht aus dem Ukrainischen, sondern aus dem Polnischen ins Deutsche übersetzt. Als Übersetzer findet sich ein gewisser G. Kanemann vermerkt, wobei es sich wohl um den auch bei Zymomrja erwähnten Kazimir Kanemann handeln dürfte, der in den Nummern 30 bis 51 des zweiten Jahrgangs 1890 auch als Übersetzer der in Fortsetzungen präsentierten Erzählung Unter dem Schutze des Gesetzes von Maria Konopnicka präsent ist.

Offensichtliche Widersprüche in der Sekundärliteratur finden sich dann in Bezug auf die Veröffentlichung von Frankos Das Märchen vom Wohlstand. Pohrebennyk führt hier den 25. November 1892 als Publikationsdatum an (Pohrebennyk 1988: 252), Zymomrja hingegen sowohl in seiner Dissertation als auch im Aufsatz des Jahres 1999 den 25. September 1892 (Zymomrja 1972: 396 bzw. Zymomrja 1999: 140). Hier stimmen nun die Angaben Pohrebennyks, der Text Frankos erschien tatsächlich in der Nummer 48 der „Arbeiter-Zeitung“ vom 25. November 1892 (48/ 25.11.1892/9-10).

Damit zu den Addenda, Texten von bzw. über Franko, die in der Sekundärliteratur meines Wissens nach bis dato noch nicht vermerkt wurden. Hier wäre zum Ersten ein lediglich mit „, $\mathrm{b}+\mathrm{i}$ “ unterzeichneter Bericht mit dem Titel Eine radikale ruthenisch-ukrainische Partei in Nummer 45 vom 7. November $1890 \mathrm{zu}$ nennen, in dem Franko positiv erwähnt wird. Es heißt hier: „Von den Namen, welche auf dem Programm unterzeichnet sind, finden wir zwei, welche in der sozialdemokratischen Partei den besten Klang haben: Iwan Franko und Michail Pawlik“ (45/7.11.1890/7). Einen bisher nicht registrierten Text Frankos, der in den Wiener Archiven zuerst von 
Jaroslav Lopušans'kyj (Drohobyč) aufgefunden wurde, bietet dann die Nummer 15 vom 8. April 1892: Hier findet sich im Unterhaltungsteil auf den Seiten 9 bis 10 Frankos Erzählung Ein Fest, eine Übersetzung von Zadlja praznyka, in der Kaiser Franz Joseph einen Auftritt hat und auf Deutsch einige Worte an die Arbeiter einer von ihm besuchten Fabrik richtet. Auch dieser Text wurde analog zu den Zigeunern nicht aus dem Ukrainischen, sondern von I. Brod aus dem Polnischen übertragen (die polnische Fassung erschien 1891, die ukrainische erst zwanzig Jahre später).

Interessant ist in diesem Kontext auch der im Original nicht vorhandene Untertitel „Eine wahre Begebenheit“", belegt dieser doch die Funktionalisierung von Literatur als Sozialdokument, die in der „Arbeiter-Zeitung“ gerade auch in Zusammenhang mit Franko mehrfach vorhanden ist. Literarische Texte werden hier stets mit einer außerästhetischen Blickrichtung als Beleg für die beklagenswerten sozialen Umstände der Arbeiterschaft sowie appellativ als Ausgangspunkt sozialen Handelns präsentiert. Als Beleg in diese Richtung kann etwa die am 25. August 1893 unter der Rubrik „Von nah und fern“ erschienene Notiz Höchster Besuch in Berndorf dienen, in der polemisch über den Besuch von Erzherzog Karl Ludwig in der Berndorfer Metallwarenfabrik und die unterwürfige Berichterstattung darüber im „Wiener Salonblatt" referiert wird (34/25.8.1893/1). Die hier referierten Einzelheiten des Besuches lesen sich wie ein faktisch verbürgter Gegenentwurf zur Erzählung Zadlja praznyka, deren fiktiver Charakter durch den zuvor erwähnten Untertitel „Eine wahre Begebenheit“ ja nach Kräften zurückgestuft wurde.

Einen in der Sekundärliteratur offensichtlich nicht vermerkten Wiederabdruck eines bereits an anderer Stelle veröffentlichten Textes von Franko bietet der Jahrgang 1895 der „Arbeiter-Zeitung“; mit der 1895 vorgenommenen Umstellung auf den Erscheinungsmodus einer Tageszeitung (zuvor erschien die „Arbeiter-Zeitung“ zweimal pro Woche jeweils dienstags und freitags) wurde der Zeitung eine Sonntagsbeilage angefügt, die eigens und durchgehend paginiert war und im Zeichen der Wochenendunterhaltung belletristische Texte, Illustrationen, Humoristisches und Rätsel enthielt. Ganz offensichtlich mündete der damit einhergehende erhöhte Bedarf an belletristischen Texten in den Rückgriff auf diesbezügliche eigene Bestände. In dieser Hinsicht lässt sich jedenfalls das Wiederauftauchen von Frankos Phantasie in Prosa Der Bahnbrecher interpretieren, die in zwei Folgen in den Beilagen vom 24. Februar und vom 3. März 1895 erschienen ist (24.2.1895/29-30 bzw. 3.3.1895/ 33-34). Titel und Autorenangabe lassen den Schluss zu, dass es sich hier um einen erstmals veröffentlichten deutschsprachigen Text Frankos handelt, fehlt doch jeglicher Verweis auf eine Quelle oder einen Übersetzer. Tatsächlich jedoch liegt hier ein als solcher nicht gekennzeichneter Rückgriff auf einen schon gedruckten Text Frankos vor - Der Bahnbrecher erschien in dieser Form bereits am 9. Juli 1887 in Nummer 29 des sozialdemokratischen Wochenblattes „Gleichheit“, das 1886 so wie drei Jahre später auch die „Arbeiter-Zeitung“ von Viktor Adler in Wien gegründet wurde. In der „Gleichheit“" ist freilich vermerkt, dass es sich bei dem Bahnbrecher um eine Übersetzung aus dem Polnischen handelte, und mit dem „Przegląd społeczny“ 
Nr. 9/1886 auch die Quelle dieser in ukrainischer Sprache erst 1900 unter dem Titel Rubač veröffentlichten Phantasie angegeben. Warum die in der "Arbeiter-Zeitung“ für gewöhnlich gepflegte Praxis, Quellen und Übersetzer eines Textes von Franko zu vermerken, hier nicht eingehalten wurde, kann nur vermutet werden - vielleicht betrachtete man das Vorgängerorgan „Gleichheit“ als ohnehin zur „Arbeiter-Zeitung" gehörig und verzichtete in der Sonntagsbeilage des Jahres 1895 deshalb auf diese Angaben.

Im Jahrgang 1896 ist am Freitag, den 27. März unter der Rubrik „Vom Tage“, mit der die „Arbeiter-Zeitung“ damals ihre Berichterstattung eröffnete, zu lesen, dass Paul Ritter von Tyszkowski am selben Tage als Folge manipulierter Wahlen zum Reichsratsabgeordneten gewählt wurde und Ivan Franko als Gegenkandidat zu Tyszkowski unterlegen sei. Das Blatt greift daran anschließend und unter Nennung der Quelle auf Frankos am 9. November 1895 in Nummer 50 der „Zeit“ veröffentlichten Bericht Die jüngste galizische Wahl zurück, aus dem teils in Paraphrase, teils im direkten Zitat über die Manipulationen der Wahl in Przemyśl, über Stimmenkauf und Drohungen berichtet wird (86/27.3.1896/1). In das Jahr 1896 fällt dann auch ein bis dato nicht registrierter Nachdruck von Frankos Erzählung Das Recht des Schweines, die im selben Jahr in der Nummer 88 der Wiener „Zeit" veröffentlicht wurde. Der 1963 edierte Band mit den deutschen Texten Frankos hält in den Anmerkungen zu dieser Erzählung mehrere Periodika fest, in denen der Text nachgedruckt wurde, darunter die "Sächsische Arbeiter-Zeitung“ und die „Münchener Post“" (Franko 1963: 544), Cieśla bzw. Nahirnyj übernehmen diese Angaben in ihren Aufsätzen (Cieśla 1976: 338 bzw. Nahirnyj 1998: 806) und Zymomrja schließlich erwähnt daneben noch etwa das „Hamburger Echo“ (Zymomrja 1999: 141). In keiner der drei Darstellungen freilich erfährt man, dass die Erzählung auch in der „Arbeiter-Zeitung" vom 10. Juni 1896 mit dem entsprechenden Vermerk „Die Zeit" am Schluss des Textes erschien (159/10.6.1896/4-5).

Die nächsten beiden Erwähnungen Ivan Frankos stehen dann wiederum in $\mathrm{Zu}$ sammenhang mit dessen politischer Tätigkeit: Am 29. September 1896 wird unter der Rubrik „Inland“ über den Parteitag der ruthenischen Radikalen in Lemberg berichtet, bei dem Franko als Einberufer die Versammlung begrüßte, zum stellvertretenden Vorsitzenden gewählt wurde und im Namen des Zentralausschusses Bericht erstattete (268/29.9.1896/3); in Fortsetzung davon wurde am 4. Oktober über Frankos Stellungnahme referiert, wonach die neugegründete ruthenische sozialdemokratische Partei ein Bindeglied zu den polnischen Sozialdemokraten wie auch zu den ruthenischen Radikalen und zwischen den „Proletariern des flachen Landes und den Proletariern der Stadt" werden könne. Franko selbst schloss den Parteitag dann mit einem Hochruf auf diese drei erwähnten Parteien (273/4.10.1896/4).

Der letzte Verweis auf Franko aus dem Jahre 1896 belegt erneut die enge indirekte Verbindung zwischen der „Arbeiter-Zeitung“ und der "Zeit“, auf die man ungeachtet der teilweise heftig geführten Polemiken gegen die "Zeit“ in der „ArbeiterZeitung" offensichtlich gerne zurückgriff, wenn es um den ukrainischen Autor ging. 
Genau am Heiligen Abend findet sich ein mit „Krakau, 18. Dezember“ datierter und mit Die galizische Bauernbewegung überschriebener Bericht des polnischen Sozialdemokraten Ignacy Daszyński, der ein Jahr später 1897 in den Reichsrat gewählt werden sollte. Der polnische Politiker übt hier Kritik an Frankos Haltung, wobei Franko eingangs als begabtester Dichter und Publizist unter seinen ruthenischen Zeitgenossen gewürdigt wird. Weiter heißt es: „Wir verübeln dem Herrn Dr. Franko nicht, dass er der polnischen Volkspartei nicht kritisch genug gegenübersteht; wir haben nämlich den Dichter und Gelehrten Franko nie für einen kritischen und klaren Politiker gehalten“. Im Anschluss daran kommt Ignacy Daszyński noch auf die „Zeit" zu sprechen, in der sich Franko mit seinem Aufsatz über die Auswanderung (gemeint ist wohl der in Nr. 115 am 12. Dezember 1896 veröffentlichte Bericht Die Auswanderungsagenten in Galizien) über das Massenelend der galizischen Bauern als gut informiert gezeigt habe (354/24.12.1896/2).

Das Jahr 1897 bietet lediglich eine einzige Referenz auf den ukrainischen Autor: Am 28. und am 29. September wird jeweils unter der Rubrik „Inland“ erneut über den Parteitag der ruthenischen Radikalen in Lemberg berichtet, den Franko als Vorsitzender des Parteivorstandes eröffnete. Daneben erfährt man noch, dass Franko ins Präsidium gewählt wurde und zu verschiedenen Punkten der Diskussion Stellung nahm (268/28.9.1897/2-3 bzw. 269/29.9.1897/4).

Wesentlich interessanter scheint die erste Erwähnung des ukrainischen Autors im Jahr 1898, konturiert sie doch analog zu Frankos in der ,Zeit“ eröffneter Auseinandersetzung mit Mickiewicz oder seiner in Wien geschlossenen Bekanntschaft mit Josef Svatopluk Machar die intensiven, teilweise auch durchaus konfliktträchtigen innerslawischen Kontakte im kulturpolitischen Rahmen der Donaumonarchie. Beginnend mit 19. Juni 1898 berichtete die „Arbeiter-Zeitung“ breit über die in Prag stattfindenden Feierlichkeiten anlässlich von František Palackýs 100. Geburtstag. Am 24. Juni erfährt man nun, dass die radikalen Ruthenen die Teilnahme an dieser Feier abgelehnt haben, wobei die Absage der ruthenischen Vereine Lembergs neben Prof. Romančuk auch von Ivan Franko unterschrieben wurde. Nun werden aus diesem Schreiben die Gründe für die Absage trotz der Hochschätzung für Palacký zitiert: Die politische Vertretung des tschechischen Volkes gehe nicht im Sinne der slawischen Idee vor, da sie den schwerwiegenden Wahlbetrug in Galizien beschönigt habe. Die Feierlichkeit könnte laut dem Schreiben nun für die politischen Anliegen dieser Vertretung ausgenützt werden, aus diesem Grunde möchte man sich auch dagegen verwehren, dass dort irgendjemand im Namen des ruthenischen Volkes spricht (172/24.6.1898/3).

Der nächste Beleg aus dem Jahre 1898 veranschaulicht wie schon einige davor die enge Verflechtung der „Arbeiter-Zeitung“ mit anderen Wiener Periodika, wie etwa der „Zeit“, aus denen entweder Beiträge direkt in das eigene Blatt übernommen wurden oder auf die man in den eigenen Berichten Bezug nahm. Am 21. August findet sich unter der Rubrik „Inland“ ein Beitrag mit der Überschrift Die Niederlage des Dr. Iwan Franko, in der über die letzten Ergänzungswahlen im Bezirk Tarnopol 
berichtet wurde, bei denen der Kandidat des polnischen Zentralwahlkomitees, Dr. Hladyszowski, mit 266 gegen Ivan Franko als dem ruthenisch-radikalen Kandidaten mit lediglich 256 Stimmen gewählt wurde. Nun wird in dem Beitrag auf die letzte Nummer der Wiener Wochenschrift „Die Wage“ verwiesen, in der Roman Sembratovyč $\check{c}^{4}$ nachgewiesen habe, wie die Manipulationen dieser Wahl (Franko erhielt tatsächlich nämlich mindestens 298 Stimmen) abgelaufen sind (229/21.8.1898/3). Bei dem erwähnten Aufsatz in der "Wage“ handelt es sich um den Text Der siegreiche Schwindel, der am 20. August 1898 in Nr. 34 der „Wage“ veröffentlicht wurde; in ebendieser Nummer finden sich daneben noch eine Würdigung Frankos anlässlich seines 25-jährigen schriftstellerischen Jubiläums aus der Feder von Saul Raphael Landau sowie Frankos Erzählung Mein Verbrechen.

Die dritte und letzte Erwähnung des Jahres 1898 führt wiederum zu Frankos politischem Engagement zurück und referiert am 12. November unter der Rubrik „Inland“ über den siebenten Parteitag der ruthenisch-radikalen Partei, der an den letzten beiden Oktobertagen 1898 in Lemberg stattfand. Franko erklärte auf dem Parteitag, dass die fanatische Wut der ruthenischen Geistlichkeit seinen Erfolg bei der Kandidatur zum Reichsrat in Tarnopol verhindert habe, und verwies darauf, dass die Popen immer und überall gegen die Partei und das Volk selbst handelten. Am Parteitag wurde daneben laut Bericht auch die Haltung der radikalen Partei zur Sozialdemokratie erläutert, wobei ein Flügel der Partei lediglich für eine kleinbäuerlich geprägte Opposition stehen wollte; die Führer dieser ,nichtsozialistischen Abteilung“ wären nun Männer wie Franko und Pavlyk (312/12.11.1898/2).

Corrigenda und Addenda gleichermaßen lassen sich schließlich auch zur bibliographisch gut dokumentierten Übersetzung von Frankos Boa constrictor in der „Arbeiter-Zeitung" beibringen. Der Text erschien in Fortsetzungen in den Nummern 47 bis 74 zwischen dem 17. Februar und dem 16. März 1901 und wurde laut Angabe des Übersetzers Karl Helbich aus dem Ruthenischen übertragen. Cieśla übernimmt nun diese Angabe in seine Darstellung und charakterisiert Helbich weiter als in Böhmen lebenden Arzt (Cieśla 1976: 337). Pohrebennyk dagegen weist korrekt darauf hin, dass die Übersetzung nicht nach dem ukrainischen Original, sondern nach der 1897 in der Reihe „Světová knihovna“ in Prag erschienenen tschechischen Übersetzung von František Hlaváček angefertigt wurde (Pohrebennyk 1988: 253). ${ }^{5}$ Dies lässt sich einmal anhand eines Briefes, den Helbich am 19. August 1901 an Franko richtete, bestätigen; Helbich erwähnt hier explizit die tschechische Übersetzung von Boa constrictor als Ausgangspunkt seiner eigenen Übertragung sowie das eigen-

${ }^{4} \mathrm{Zu}$ diesem zentralen Vertreter des ukrainischen Geisteslebens in Wien, dessen wichtigste Leistung die Herausgabe der 1903 in Wien gegründeten „Ruthenischen Revue“ gewesen ist, vgl. Lopušans'kyj 1995.

51897 als Jahr der Veröffentlichung ist aus den Angaben Pohrebennyks übernommen, das von mir in der Österreichischen Nationalbibliothek eingesehene Exemplar der Übersetzung ist undatiert und trägt die handschriftlich hinzugefügte Datierung 1898. 
mächtige Vorgehen der Redaktion der „Arbeiter-Zeitung“, die trotz seines ausdrücklichen Verbots der Übersetzung die Bemerkung „Aus dem Ruthenischen übersetzt“ vorangestellt hatte (Franko 1963: 485 f.).

Daneben vermag aber auch eine vergleichende Analyse der beiden Übersetzungen eindeutig zu belegen, dass nicht die ukrainische Erstfassung, sondern die tschechische Übersetzung Hlaváčeks der Ausgangspunkt für Helbichs Übertragung gewesen ist. Dies geht bereits aus dem ersten Satz des Textes hervor, der bei Helbich „Hermann Goldkrämer erhob sich erzürnt“ (47/17.2.1901/ 8), im ukrainischen Original hingegen „Herman Gol'dkremer vstav nyni duže zlyj“ (Franko 1978: 370) lautet. Der Verlust der Zeitbestimmung „nyni“ erklärt sich aus der tschechischen Übertragung „Hermann Goldkremer vstal velmi rozzloben“ (Franko 1897: 3). Ebenso sind die „Arbeiter“ im übernächsten Satz aus der tschechischen Fassung (dort als „,ělníkům"), nicht hingegen aus der ukrainischen herzuleiten, wo etwas enger gefasst von „ripnykam“, also den Arbeitern in den Naphtagruben, die Rede ist. Und noch ein letzter Beleg: Die Stimme der Arbeiter bei Helbich (,Und ihre Stimme ...") findet ihre Vorlage in der tschechischen Zwischenstufe (,A jejich hlas“ [4]), nicht hingegen im ukrainischen Original, wo die Stimmen in den Plural gesetzt sind („I holosy u nych").

$\mathrm{Zu}$ beachten ist neben der Erzählung selbst auch deren Ankündigung, die am 16. Februar 1901 - also einen Tag, bevor die erste Folge des Textes erschien - in der „Arbeiter-Zeitung“ veröffentlicht wurde. Von Interesse ist an dieser Anzeige, die laut Zymomrja wahrscheinlich von Viktor Adler selbst verfasst wurde (Zymomrja 1972: 401) und mit An unsere Leser! überschrieben ist, einmal die äußerst prominente Platzierung im Blatt selbst - sie findet sich nämlich auf Seite eins in Nummer 46 ganz links oben, also unmittelbar am Anfang der Nummer. Analog zu dieser Hervorhebung erfolgt auch die Präsentation Ivan Frankos, der hier als bereits gut eingeführte literarische Größe vorgestellt wird: „Schon der Name des Verfassers, den viele unserer Leser sehr gut kennen, enthebt uns eigentlich der Aufgabe, die ebenso gehaltvolle wie spannende Erzählung noch besonders zu empfehlen" (46/16.2.1901/1).

Des Weiteren tritt wie schon im Kontext mit der Erzählung Zadlja praznyka auch hier deutlich die Dominanz außerästhetischer Wertungsmaßstäbe von Literatur zu Tage. Die Wahrnehmung von fiktiven Texten als Sozialdokument findet sich dabei in diesem Falle in den vorangegangenen Jahrgängen der "Arbeiter-Zeitung“ gleichsam sukzessive vorbereitet. Mehrmals wird in Berichten und Notizen in sehr emotional gehaltenen und einander ähnlichen Worten die soziale Not der Grubenarbeiter im galizischen Boryslav, wo Frankos Text ja spielt, angeprangert, so erstmals am 5. Februar 1892 in einem Bericht zum Kongress der Sozialdemokratie Galiziens, wo ein Arbeiter aus Boryslav über die Arbeitsbedingungen in den dortigen Gruben berichtet:

Die Wohnungsverhältnisse spotten jeder Beschreibung, es sind dies schmutzige finstere Schoppen in denen Männer, Frauen und Kinder pêle-mêle durcheinander liegen. Die moralischen Verhältnisse sind auch darnach. Es ist nicht selten, daß Bruder und Schwester 
geschlechtlichen Verkehr pflegen. [...] Um die Witwen und Waisen der Verstorbenen oder Verunglückten kümmert sich Niemand, sie sind ihrem Schicksal preisgegeben.

$(6 / 5.2 .1892 / 4)$

Ein Jahr später liest man dann am 22. Dezember 1893 in der Rubrik „Von nah und fern“: „Zwölftausend Proletarier - Sklaven, welche in den Boryslawer Bergwerken arbeiten, wurden zu dem Niveau einer solchen thierischen Verrohung, eines solchen beispiellosen Elends niedergedrückt, daß in ganz Österreich kein Bergwerksoder Fabriksort damit verglichen werden kann“" (59/22.12.1893/2). Ein ähnliches, das Elend und die Ausbeutung in Boryslav anklagendes Bild zeichnen auch die Berichte vom 4. August 1895 über die Zustände ebendort (211/4.8.1895/3) sowie vom 3. November 1895 zum Naphtabergbau in Galizien (302/3.11.1895/3-4).

All diese Zeugnisse nichtfiktionaler Natur konstituieren gleichsam die Bedingungen, unter denen dann schließlich Frankos Boa constrictor am 16. Februar 1901 präsentiert wird. Vor dem Hintergrund des in der „Arbeiter-Zeitung“ immer wieder thematisierten Elends der Arbeiter in Galizien wird dem literarischen Text in erster Linie Relevanz als Sozialdokument und dann erst als Kunstwerk zugesprochen, wenn es heißt:

Die Erzählung spielt in den Gegenden der Naphtagruben von Boryslaw und schildert die soziale Lage der fürchterlich ausgebeuteten Arbeiterschaft. Schon dieses Milieu sichert der Erzählung unser besonderes Interesse, abgesehen von der großen Kunst und Darstellung, über die der einsame ruthenische Dichter in so großem Maße verfügt.

$(46 / 16.2 .1901 / 1)$

Auch hier tritt ähnlich wie schon bei der Übersetzung von Zadlja praznyka das Bestreben des Blattes hervor, Literatur mit den Maßstäben einer als sozial veränderbar konzipierten Realität zu messen und deren Autonomieanspruch an die zweite Stelle zu rücken, wie die zuvor zitierte Formulierung ,abgesehen von der großen Kunst und Darstellung" deutlich erkennen lässt. Dass Ivan Franko mit seiner realistischen Darstellung galizischer Lebenswelten bzw. der im Bahnbrecher zu beobachtenden Allegorisierung einer besseren, der Selbstaufopferung werten Zukunft gut in dieses Schema hineinpasste, mag wohl die kontinuierliche Präsenz seiner Texte in der „Arbeiter-Zeitung“ erklären.

Abschließend soll noch ein knapp gehaltener Verweis auf einen scheinbar marginalen Text folgen, der aber schlaglichtartig jene Hierarchisierung verschiedener Kulturen aufzuzeigen vermag, die letztlich auch in der hier referierten „Arbeiter-Zeitung" zu beobachten ist: Am 28. Juli 1893 findet sich inmitten der Kleinanzeigen ein Aufruf zu einer polnischen Volksversammlung in Hambergers Saal „Zur blauen Weintraube“ im fünften Wiener Gemeindebezirk, bei der die Referate in polnischer, ruthenischer und deutscher Sprache gehalten wurden (30/ 28.7.1893/6). Ganz offensichtlich nimmt der Begriff ,polnisch“ hier eine privilegierte Position ein, die auch den ukrainischen Bereich mitumfasst. Analog dazu ist Franko auch in der ,ArbeiterZeitung" über die Übersetzungen seiner Texte entweder als polnischer oder mit sei- 
nen Originalbeiträgen als österreichischer Autor, aber eigentlich nicht als ukrainischer Schriftsteller präsent - die angebliche Übertragung von Boa constrictor aus dem Ukrainischen erfolgte tatsächlich ja aus dem Tschechischen. Als ukrainischer Autor im eigentlichen Sinne sollte Franko erst in der bereits erwähnten, ebenfalls in Wien situierten „Ruthenischen Revue“ (späterhin als „Ukrainische Rundschau“) eigenständiges Profil gewinnen. Dessen ungeachtet kam der Wiener „Arbeiter-Zeitung" dank ihrer regelmäßigen Berichterstattung zu Ivan Franko sowie durch die ebenso regelmäßig erfolgte Veröffentlichung seiner Beiträge neben anderen deutschsprachigen Periodika jener Zeit eine nicht zu unterschätzende Bedeutung bei der sukzessiven Popularisierung des ukrainischen Schriftstellers zu. Ein zumindest teilweises, die bereits existierende Sekundärliteratur zur gegebenen Thematik ergänzendes und korrigierendes Nachzeichnen dieser von der „Arbeiter-Zeitung“ übernommenen Funktion sollte der hier vorliegende Beitrag geboten haben.

\section{L it e ratur}

Bendzar 1966:

Bendzar 1969:

Cieśla 1976:

Franko [1897]:

Franko 1963:

Franko 1966:

Franko 1978:

Lopušans'kyj 1995:

Machar 1902:

Magocsi 1979/80:

Nahirnyj 1998:

Pohrebennyk 1988:

Simonek 1993:

Wytrzens 1991:

Zymomrja 1972:
B. Bendzar, Publicystični tvory Ivana Franka nimec'koju movoju, Dnipro 8/1966, 136-141

B. P. Bendzar, Tvorčestvo Ivana Franko na nemeckom jazyke. Avtoreferat dissertacii na soiskanie učenoj stepeni kandidata filologičeskich nauk, Kiev

M. Cieśla, Niemiecka oryginalna twórczość literacka Iwana Franki, Slavia Orientalis 25, 333-342

I. Franko, Boa constrictor. Autorisovaný překlad z maloruštiny od Františka Hlaváčka, Praha, o. J.

I. Franko, Beiträge zur Geschichte und Kultur der Ukraine. Ausgewählte deutsche Schriften des revolutionären Demokraten 1882-1915, hg. v. E. Winter u. P. Kirchner, Berlin-Ost

I. Franko, Bibliohrafija tvoriv 1874-1964, Kyjiv

I. Franko, Zibrannja tvoriv t. 14, Kyjiv

Ja. Lopušans'kyj, Roman Sembratovyč u konteksti jevropejizaciji ukrajins'koji kul'tury, Acta Philologica III, Drohobyč, 8-16

J. S. Machar, Knihy feuilletonů. Druhá: 1896-1900, Praha

P. R. Magocsi, Vienna as a Resource for Ukrainian Studies: With Special Reference to Galicia, Havard Ukrainian Studies III/IV, 609-626

M. Nahyrnyj: Ivan Franko jak učasnyk istoryko-literaturnoho procesu Avstriji ta Nimeččyny, in: Ivan Franko - pys'mennyk, myslytel', hromadjanyn. Materialy Mižnarodnoji naukovoji konferenciji (L'viv, 2527 veresnja 1996 r.), L'viv, 801-807

Ja. M. Pohrebennyk, Ukrajins'ko-nimec'ko-avstrijs'ki literaturni zv'jazky XIX - počatku XX st., in: Ukrajins'ka literatura v zahal'noslov'jans'komu i svitovomu literaturnomu konteksti v 5-y tomach. T. 3: U vzajemynach z literaturamy zachodu i schodu, Kyjiv, 220-276

S. Simonek, Drei Blicke auf Wien: I. Franko - J. S. Machar - I. Cankar. Eine komparatistische Skizze, Wiener Slavistisches Jahrbuch 39, 131-143

G. Wytrzens, Zum literarischen Schaffen Ivan Frankos in deutscher Sprache, Wiener Slavistisches Jahrbuch 37, 103-112

N. I. Symomrja, Die Rezeption ukrainischen Literaturgutes im deutschen Sprachgebiet von den Anfängen bis zur Großen Sozialistischen Oktoberrevolution von 1917. Ein Beitrag zur Geschichte der ukrai- 
nisch-russisch-deutschen Literaturbeziehungen, Berlin-Ost [Dissertation]

Zymomrja 1999: M. Zymomrja, Übersetzungskritik und ihre Stellung im Rezeptionsprozeß (Entwicklungstendenzen der Aufnahme von I. Frankos Werken in Deutschland), in: Ders., Deutschland und Ukraine: Durch die Abrisse zur Wechselseitigkeit von Kulturen. Forschungsbeiträge, hg. v. I. Tratsch, Fürth/Bayern, 135-142

\author{
Stefan Simonek \\ Institut für Slawistik der Universität Wien \\ Universitätscampus AAKH, Hof 3 \\ Spitalgasse 2, A-1090 Wien \\ stefan.simonek@univie.ac.at
}

